enthusiasm for the speciality, was a key factor in the creation of a holistic, AYA-focussed ethos of care. Centralising AYA cancer services would increase the experience HCPs have of working with AYA, within age-appropriate environments of care, thus fostering an ethos of care sensitive to AYA holistic needs. Whether this impacts outcome will become evident in early 2019 when the results of BRIGHTLIGHT are released.

\section{USING QUALITATIVE SYNTHESIS DATA TO INFORM INTERVENTIONS IN PALLIATIVE CARE: A MULTILEVEL APPROACH}

${ }^{1}$ Megan Armstrong, ${ }^{2}$ Kate Flemming, ${ }^{1}$ Nuriye Kupeli, 'Patrick Stone, ${ }^{3}$ Susie Wilkinson, ${ }^{1}$ Bridget Candy. 'University College London, London, UK; ${ }^{2}$ University of York, York, UK; ${ }^{3}$ University of Liverpool, Liverpool, UK

\subsection{6/bmjopen-2019-QHRN.25}

Introduction Complex interventions are common in palliative care (PC), but the data from quantitative and qualitative research can be contrasting. For example, systematic reviews found trial evidence on effectiveness was uncertain in complementary therapies (CTs) in PC; however, based on qualitative evidence patients' perceived CT to be highly beneficial for their well-being and highlighted ways in which they wished CT were delivered.

Aim To develop an exemplar in PC that draws together the findings from quantitative and qualitative systematic reviews to inform reasons for discrepancies between the two and suggest directions for future intervention development.

Methods We sought trials on the effectiveness of CT and qualitative studies on patients' perspectives about CTs. Our primary outcomes for trials included anxiety, pain, and quality of life. Eight databases were searched in 2018. Citations and fulltext papers were reviewed independently to identify relevant studies. Meta-analyses to pool trial data were considered and a thematic synthesis was conducted to understand patients' experiences as presented in primary qualitative analysis. The individual review findings were combined in matrices to explore similarities and differences.

Results Twenty-two trials and five qualitative studies were included. A matrix table explored the (lack of) overlap between items on a commonly used quality of life measure from the review of trials and the themes from the thematic synthesis. A table was also created to explore the variations between how patients want CT to be delivered and how it is being delivered in trials.

Conclusions This combining of qualitative and quantitative data has highlighted outcome measures in trials may be inappropriate and the interventions may not be delivered how patients wish. Our approach demonstrates a potential way in PC to enhance development of practice appropriate complex interventions.

\section{O26 OPTIMAL INVOLVEMENT OF PATIENTS IN THE MORBIDITY AND MORTALITY MEETING (OPTIMA STUDY)}

Britt Myren, Rosella Hermens, Joanne De Hullu, Leon Massuger, Petra Zusterzeel. Radboud University Medical Center, Nijmegen, The Netherlands

10.1136/bmjopen-2019-QHRN.26
Background Different research has suggested that involving patients during meetings, normally intended for doctors, is beneficial: not only to inform patients, but also to integrate the patient's perspective in the discussions on future cure and care. However, inviting the patient to join such a meeting, is a new field. In general, surgical departments worldwide hold a Morbidity and Mortality ( $\mathrm{M}$ and $\mathrm{M}$ ) meeting to discuss medical errors, (unexpected) adverse events or near misses. The healthcare professionals discuss what happened, why and what they can do to prevent this in the future. It can be challenging to discuss this openly in front of the patient.

In 2016 a pilot has been started to invite the patient to join the monthly $\mathrm{M}$ and $\mathrm{M}$ meeting at the department of gynecological oncology (Radboudumc, Nijmegen, the Netherlands).

We evaluated the $M$ and $M$ meetings with patient participation since its pilot. What are the burdens and benefits for patients and healthcare professionals, in order to improve the $\mathrm{M}$ and $\mathrm{M}$ meeting?

Methods We conducted in-depth interviews with 10 patients and 15-20 healthcare professionals. Two $\mathrm{M}$ and $\mathrm{M}$ meetings were observed. The analyses were done by thematic coding using the program Atlas.ti, but framed in order to choose next methodological steps and ways to implement this format in other departments.

Preliminary results Patients feel safe to share their experience during the discussion and feel fully informed. Healthcare professionals gain new insights from the patient and their partners' perspective. However, it is more difficult to choose the right words and communicate open and honest during the discussion.

Conclusion The current $M$ and $M$ meeting format with patient participation is improved and next methodological steps are chosen.

\section{O27 KNOWLEDGE-TO-ACTION: PROMOTING BEST PRACTICES FOR MATERNAL OPIOID USE}

Tracy Nichols, Meredith Gringle. University of North Carolina Greensboro, Greensboro, USA

\subsection{6/bmjopen-2019-QHRN.27}

Background An increase in opioid use during pregnancy fuelled concerns among reproductive health advocates, policymakers, and providers in North Carolina, USA. Stakeholder groups joined together to address these concerns. A knowledge transfer (KT) intervention, which provided specialized education and training for frontline providers, in an effort to increase evidence-based practices became the primary goal of their response. Qualitative methodology can provide critical insight on the process of KT interventions and the contexts in which KT activities occur.

Methods Data from a grounded theory study on care provision for perinatal substance use was used to examine a naturalistic KT intervention that emerged from stakeholder concerns. Data was collected over a six-year period and include observations of KT activities (conferences, workshops, and community meetings), focus groups and interviews conducted with stakeholders, and a review of publicly available documents developed as part of KT activities. Identified KT activities were mapped onto a theoretical framework detailing the Knowledge-to-Action (KTA) process and a thick, rich description of the intervention was developed. Additional 
analyses included Clarke's situational mapping to identify important contextual factors that influenced the KTA process. Findings This proactive response to maternal opioid use included targeted and tailored toolkits and guidelines of best practices as well as provision of multiple knowledge exchange opportunities between researchers, advocates, and practitioners. Findings demonstrate stakeholders' biases against maternal drug use and ambivalence towards evidence-based recommendations of harm reduction principles. Tensions around the interpretation of knowledge and the appropriate implementation of best practices occurred between 'experts' as well as among practitioners.

Conclusion This presentation describes a grassroots response to maternal opioid use within the KTA framework. Findings highlight the complex and iterative nature of a KTA intervention and demonstrate limitations in rational and cognitive approaches to knowledge exchange. The need to systematically address stigma is discussed.

\section{Parallel session - Ethnographic Encounters (22 March 14:00 -15:15)}

\section{O28 NEOLIBERAL POLICY REFORMS \& BIOMEDICAL RESIDUALISM IN COMMUNITY MENTAL HEALTH PRACTICE IN ENGLAND}

Rich Moth. Liverpool Hope University, Liverpool, UK

\subsection{6/bmjopen-2019-QHRN.28}

Background Mental health policy initiatives in England over the last three decades have led to significant restructuring of statutory service provision. One feature of this has been the reconfiguration of NHS mental health services to align with the requirements of internal and external markets in the context of wider neoliberal organisational transformations.

Aims/Objectives This paper examines the effects of neoliberal policy and service reforms on professional practice and conceptualisations of mental distress within one mainstay of NHS statutory services: the community mental health team (CMHT).

Methods The paper is based on findings from twelve months' ethnographic fieldwork and semi-structured interviews with practitioners and service users within two CMHTs.

Results and conclusions The paper begins with an account of the restructuring of the labour process in mental health services. This utilises the notion of 'strenuous welfarism' ${ }^{1}$ to describe an organisational context characterised by escalating performance management, deskilling of professional practice and the intensification of mental health work. The study found that increasingly prominent aspects of managerialism and marketization disrupted attempts by mental health practitioners to sustain supportive and mutual structures with colleagues and engage with service users in therapeutic and relationship-based forms of practice. Moreover organisational processes increasingly recast service users as individual consumers 'responsibilised' to manage their own risk, or subject to increasingly coercive measures when perceived to have failed to do so. Consequently biomedical orientations were remobilised in practice in spite of a rhetorical shift in policy discourse towards socially inclusive approaches. The term 'biomedical residualism' is coined to describe this phenomenon.
However instances of ethical professionalism that reflected resistance to these residualised modes of practice were also visible.

\section{REFERENCE}

1. Mooney G and Law A. New Labour/hard labour?: restructuring and resistance inside the welfare industry. Policy Press, 2007

\section{O29 EXPLORING THE NATURE OF HOME CARE FOR PEOPLE LIVING WITH DEMENTIA THROUGH ETHNOGRAPHIC OBSERVATIONS}

Monica Leverton. UCL, London, UK

\subsection{6/bmjopen-2019-QHRN.29}

In the UK, over 850000 people are living with a diagnosis of dementia. Many of these people wish to remain living in their own homes which is often made possible by the support of family carers and paid home care workers. However, home care is a sector that has come under much scrutiny and the workforce is often criticised for providing unsatisfactory care ${ }^{1}$. At an organisational level there is a high turnover of home care workers who are typically low-paid and receive little training and support ${ }^{2}$. We have drawn from an ethnographic approach in order to explore the nature of home care firsthand and to develop an understanding of the knowledge and training needs of home care workers. This has involved participant-observations with eight home carers across six varied locations in England, as they provide home care for clients living with dementia. As a participant-observer, we have attempted to become immersed in the reality of home care as it is experienced in the real world for those involved. Qualitative observations have enabled us to go beyond the understanding gained through interviews in order to capture the everyday experience of dementia home care. In this presentation, we will discuss the experience of conducting ethnography in a complex social care setting, faced with challenges of observing in the context of scrutiny and mistrust. The process of engaging home carers and consenting people living with dementia will be shared in order to promote the use of qualitative observations as a way of gaining a more meaningful understanding of health and social care issues within the home care workforce. As part of a wider study, these findings aim to inform the intervention development of a training programme for paid home carers, to aid consistency and accessibility of training amongst the homecare workforce.

\section{REFERENCES}

1. Muntaner $C$, et al. County and organizational predictors of depression symptoms among low-income nursing assistants in the USA. Sci Med 2006;63(6):14541465

2. Livingston $\mathrm{G}$, et al. Making decisions for people with dementia who lack capacity: qualitative study of family carers in UK. BMJ 2010;341:c4184

\section{A WEB-BASED ETHNOGRAPHIC STUDY OF PSYCHOSTIMULANT USE: A NOVEL APPROACH FOR PUBLIC HEALTH RESEARCH}

Caroline Robitaille. Université de Montréal, Montréal, Canada

10.1136/bmjopen-2019-QHRN.30 